\title{
ANDES

\section{Función pulmonar evolutiva evaluada por oscilometría de impulso en prescolares con asma}

\author{
Evolutionary lung function evaluated by impulse oscillometry in preschoolers \\ with asthma
}

\author{
Ramiro González Vera ${ }^{\mathrm{a}}$, Mónica Saavedra Bentjerodt ${ }^{\mathrm{a}}$, Alberto Vidal Grella ${ }^{\mathrm{a}}$, Jorge Mackenney Poblete ${ }^{\mathrm{a}}$
}

aCentro de Enfermedades Respiratorias Pediátricas, Clínica Las Condes. Santiago, Chile

Recibido: 15 de enero de 2020; Aceptado: 13 de septiembre de 2020

¿Qué se sabe del tema que trata este estudio?

La oscilometría de impulso (IOS) permite evaluar la función pulmonar en preescolares asmáticos y medir la respuesta broncodilatadora.
¿Qué aporta este estudio a lo ya conocido?

La evolución de la IOS permitiría caracterizar mejor la enfermedad en esta etapa de la vida, identificar a quienes su función pulmonar no mejora, en especial parámetros de la vía aérea periférica para optimizar su manejo.

\section{Resumen}

La oscilometría de impulso (IOS) es útil para evaluar la función pulmonar en preescolares. Nuestro objetivo fue describir las alteraciones y perfil evolutivo de la IOS en asmáticos menores de 6 años luego de un año de seguimiento. Pacientes y Método: 62 preescolares realizaron IOS al inicio del estudio y luego de un año. Se comparó la proporción de IOS alterada y respuesta broncodilatadora (RB+) en ambos tiempos, además de subanálisis según control de asma y presencia de atopía. Para el análisis estadístico se utilizó $\chi^{2}$ de McNemar y t-student con error $\alpha$ de 5\%. Resultados: La IOS inicial resultó alterada en el $80,6 \%$ y en el $64,5 \% \%$ luego de un año $(\mathrm{p}=0,04)$. Se encontró $\mathrm{RB}+$ en $77,4 \%$, de los niños al inicio y $83,9 \%$ al año. El grupo de asma no controlada registró mejoría significativa en los promedios X5 y D5-20, la que no fue encontrada en los asmáticos controlados. En atópicos, sólo los asmáticos no controlados mejoraron X5, AX y D5-20. Conclusión: La IOS muestra alteraciones en un alto porcentaje de preescolares con asma no controlada, lo que disminuye en forma significativa al año, pero se mantiene alterada y con $\mathrm{RB}+$ en la mayoría de ellos. Se requieren estudios adicionales para identificar distintos fenotipos de asma del prescolar y su evolución con el tratamiento.
Palabras clave: Oscilometría de Impulso; Función Pulmonar; Asma Bronquial; Atopía; Broncodilatadores; Preescolares

Correspondencia:

Alberto Vidal

aevgmd@yahoo.es 


\begin{abstract}
Impulse oscillometry (IOS) is useful for measuring lung function in preschool children. Our objective was to describe the alterations and evolutionary profile of IOS in asthmatic children under 6 years of age after one year of follow-up. Patients and Method: 62 preschoolers performed IOS at the beginning of the study and after one year. The proportion of altered IOS and bronchodilator response (BR $+)$ at both times was compared, in addition to sub-analysis according to asthma control and presence of atopy. For the statistical analysis, we used McNemar's $\chi^{2}$ and the Student's t-test with a 5\% $\alpha$ error. Results: The initial IOS was altered in $80.6 \%$ and in $64.5 \% \%$ after one year $(p=0.04) .77 .4 \%$ of the children presented BR+ at the beginning of the study and $83.9 \%$ after one year. The uncontrolled asthma group presented a significant improvement in the X5 and D5-20 means, but the controlled asthma group did not. In atopic patients, only uncontrolled asthmatics improved X5, AX, and D5-20. Conclusion: IOS shows alterations in a high percentage of preschoolers with uncontrolled asthma, which decreases significantly at one year, but remains altered and with BR + in most children. Additional studies are required to identify different preschool asthma phenotypes and their evolution with treatment.
\end{abstract}

Keywords:

Impulse Oscillometry;

Preschool

Children; Asthma;

Lung Function;

Bronchodilators; Atopy

\section{Introducción}

El asma en prescolares es un problema común. Se han descrito prevalencias que van desde 6 a $18 \%{ }^{1}$. Muchos de esos niños continuarán con síntomas a edades mayores, especialmente aquellos con cuadros más severos y algunos presentarán función pulmonar disminuida en la adolescencia ${ }^{2-4}$. Evaluar la función pulmonar a esta edad es fundamental para realizar un diagnóstico y seguimiento adecuado de la enfermedad. Se ha recomendado utilizar la espirometría adaptada para preescolares, pero un porcentaje de los niños no logra realizar un examen que cumpla con los criterios de calidad ${ }^{5,6}$. En este escenario, la oscilometría de impulso (IOS) surge como una buena alternativa, ya que es una técnica que no requiere de una maniobra espiratoria forzada y se realiza durante la respiración a volumen corriente, en un corto periodo de tiempo. Se ha informado que distingue niños preescolares asmáticos de normales, con mayor sensibilidad que la espirometría ${ }^{7}$. En IOS, R5 refleja la resistencia de la vía aérea total, $\mathrm{R} 20$ la vía aérea proximal, $\mathrm{X} 5$ es la reactancia de la vía aérea, mide las fuerzas del movimiento de la columna de aire (inertancia) y propiedades elásticas del pulmón (capacitancia). AX integra la reactancia en todas las frecuencias y en conjunto con R5-20 son los parámetros más sensibles para detectar cambios en la vía aérea periférica ${ }^{8}$. En el último tiempo se ha dado importancia a los parámetros AX, R5-R20 y R5-20\%, ya que han mostrado ser útiles para detectar alteraciones de la función pulmonar en niños en edad preescolar y reflejarían las alteraciones en la función de la vía respiratoria pequeña, con mayor sensibilidad que los parámetros espirométricos ${ }^{9,10}$. La evaluación de la vía aérea periférica es especialmente importante porque se ha relacionado su disfunción con la pérdida del control del asma ${ }^{11}$. Se ha informado que la IOS es útil para predecir exacerbaciones de asma en niños pequeños y en el monitoreo de la enfermedad a mediano y largo plazo $^{12-14}$. La IOS es un examen que también permite evaluar la respuesta broncodilatadora, la que permite distinguir niños sanos de asmáticos y también sería importante para el seguimiento de la enfermedad ${ }^{15,16}$. La evidencia respecto al monitoreo del tratamiento con IOS de preescolares es escasa y en nuestro medio no conocemos estudios al respecto. El objetivo de este estudio fue evaluar la utilidad de la IOS en el seguimiento de preescolares con asma no controlada, mediante la medición de su variación en un periodo de un año.

\section{Pacientes y Método}

Estudio prospectivo realizado en Clínica Las Condes, Santiago de Chile, entre agosto de 2016 y septiembre de 2018. Correspondió a una cohorte de niños asmáticos persistentes de 3 a 5 años, que concurrieron de forma no aleatoria a realizar un examen de función pulmonar en el Departamento de Neumología Pediátrica. El criterio de inclusión fue el diagnóstico confirmado de asma por un neumólogo pediatra, según las recomendaciones de la Global Initiative for Asthma (GINA) 2016 ${ }^{17}$, que requirieran tratamiento farmacológico permanente y presentaran características clínicas de asma no controlada. Se definió asma no controlada cuando los pacientes registraron tres o más episodios de sibilancias confirmadas por médico en los últimos 6 meses, y haber recibido en alguno de estos episodios tratamiento con corticoides orales por 3 a 5 días. Se excluyó a los pacientes que tenían otra enfermedad respiratoria crónica, cardíaca o neuromuscular y a aquellos con enfermedades que impidieran la ejecución de los exámenes de función pulmonar. $\mathrm{Al}$ inicio 
del estudio se realizó la IOS, denominada IOS inicial, quedando los pacientes citados para un test cutáneo. Los participantes mantuvieron controles con su médico tratante, quien indicó y/o ajustó el tratamiento. Después de transcurrido un año, se repitió la IOS, denominada IOS final. En la segunda visita fueron registrados los síntomas respiratorios, el uso de broncodilatador, corticosteroides orales, visitas de emergencia, hospitalizaciones relacionadas con el asma y el tratamiento farmacológico permanente utilizado en los últimos 3 meses. En base a estos antecedentes se clasificó a los pacientes en controlados y no controlados. Se catalogó como asmáticos no controlados a los pacientes que en el último trimestre del seguimiento habían presentado un episodio de sibilancias y/o recibido corticoides orales por 3 a 5 días y/o requerido una consulta de emergencia o ambulatoria no programada, y/o recibieran salbutamol más de 7 días por mes. Los asmáticos controlados fueron aquellos que no presentaron ninguna de las características mencionadas.

La función pulmonar se evaluó mediante un espirómetro asociado a IOS con neumotacógrafo computarizado Jaeger Viasys D-97204 modelo Master Screen IOS 732595 de fabricación alemana el año 2009, previamente calibrado según recomendaciones del fabricante. Los exámenes fueron realizados con el paciente en posición sentada, con pinza nasal, sujetando sus mejillas y respirando tranquilamente a través de la boquilla conectada al equipo, asistidos por un técnico capacitado. Los registros se hicieron al menos por 30 segundos, hasta obtener 3 lecturas sinusoidales, técnicamente aceptables, sin artefactos ni fugas. Se eligieron las pruebas con la mejor coherencia a frecuencias de 5 a $30 \mathrm{~Hz}$. La coherencia fue 0,6 a $5 \mathrm{~Hz}$ y de 0,9 a $10 \mathrm{~Hz}$. con una variabilidad entre las mediciones menores al $10 \%$ en frecuencias mayores a $5 \mathrm{~Hz}^{18}$. Los pacientes no debían tener síntomas respiratorios en las últimas 2 semanas previas a la fecha de la IOS. Se les indicó suspender el uso de broncodilatadores de acción corta la noche anterior a la prueba y los de acción prolongada 48 horas antes. Para la prueba broncodilatadora se administraron $400 \mu \mathrm{g}$ de salbutamol ( 4 inhalaciones de inhalador presurizado) en inhalador de dosis medida separadas por al menos 60 segundos a través de una aerocámara valvulada. Se registraron las medidas de resistencia $\mathrm{R} 5$ y $\mathrm{R} 20$, reactancia a $5 \mathrm{~Hz}$ (X5) y área de reactancia (AX), el registro de valores se expresó en $\mathrm{KPa} / \mathrm{Ls}$. Se calculó la diferencia absoluta entre R5 y R20 (D5-20). La IOS basal se consideró anormal si se encontró al menos una de las siguientes alteraciones: R5 $\geq 140 \%$ del predictivo $y / 0 \mathrm{X} 5 \geq 140 \%$ del predictivo y/o AX $\geq 3 \mathrm{KPa} / \mathrm{Ls}$ y/o D5-20 $\geq 0,2 \mathrm{Kpa} /$ Ls. Los valores de referencia utilizados fueron los publicados por Duiverman proporcionados por el equipo y aplicables a niños de 2,3 a 12,5 años ${ }^{19}$.
Se consideró respuesta broncodilatadora $(\mathrm{RB}+)$ cuando se encontró al menos uno de los siguientes cambios: disminución de $40 \%$ en AX, 20\% en R5 y/o R20 y/o D5-20 y/o aumento del 30\% de X5 después de dar 400 mcg de salbutamol $1^{7,10,20,21}$.

El test cutáneo (TC), se realizó según la técnica del laboratorio del centro de alergias de Clínica Las Condes con indicación de no haber recibido tratamiento con antihistamínicos o corticoides orales en los 7 días previos a la fecha del examen. Este incluyó la medición de 20 de aeroalergenos comunes certificados por el laboratorio por método Prik-Test (6 intradomiciliarios y 14 extradomiciliarios) y se consideró positivo si el diámetro de la pápula fue $3 \mathrm{~mm}$ o más, para al menos uno de ellos.

Para el análisis de los resultados se calcularon frecuencias para las variables cualitativas (proporciones) y medidas de resumen para las variables cuantitativas (promedios). Se realizó la comparación de McNemar para medir la variación efectiva de casos discordantes (positivo a negativo o negativo a positivo), entre de la IOS inicial y final, considerando a cada uno de los 62 pacientes como su propio control. Para medir la variación en promedios de valores basales y de respuesta broncodilatadora entre la IOS inicial y final se utilizó la prueba $t$ de Student para muestras dependientes o emparejadas. Esta prueba también fue utilizada en dos subanálisis con un menor grupo de pacientes. El primero se realizó para medir la variación de promedios de valores basales entre la IOS inicial y final en las dos categorías de control de asma. El segundo fue la variación de los promedios basales de entre la IOS inicial y final según el control de asma y el grado de sensibilización alérgica. Se verificó la distribución de la normalidad para las variables cuantitativas. El tamaño muestral se calculó en base a una muestra piloto. En dicha muestra se consideró necesario un mínimo de 59 pacientes para obtener una diferencia de al menos 15\% en los casos discordantes de IOS alterada entre ambos estudios y un mínimo de 62 pacientes para obtener una diferencia de al menos $0,08 \mathrm{Kpa} / \mathrm{Ls}$ entre el promedio del parámetro R5 de la IOS final respecto de la inicial, considerando un porcentaje de pérdidas no superior al 10\% para ambos cálculos. En ambas comparaciones el poder fue de $80 \%$ y el error $\alpha$ de $5 \%$. La investigación contó con la aprobación del comité de ética de la institución y se obtuvo consentimiento informado por parte de los padres.

\section{Resultados}

De los 83 pacientes invitados a participar en el estudio 11 no aceptaron y 10 presentaron criterios de exclusión, por lo tanto, se enrolaron 62 pacientes. En 
la tabla 1 se muestran los datos demográficos de los pacientes. Al inicio del estudio todos los pacientes recibían tratamiento farmacológico permanente para su asma. En los últimos 3 meses del seguimiento 61 de los 62 pacientes $(98,3 \%)$ mantuvieron algún tipo de tratamiento controlador, $46(74,2 \%)$ recibía corticoides inhalados, $6(9,7 \%)$ montelukast, $5(8,1 \%)$ corticoides inhalados más montelukast y $4(6,5 \%)$ beta 2 agonista de acción prolongada más corticoide inhalado. No hubo pérdidas, ya que la totalidad de los pacientes enrolados fueron capaces de realizar la IOS final y completar el seguimiento al año. En los últimos 3 meses de seguimiento se clasificaron como controlados a 28 pacientes $(45,2 \%)$ y no controlados a 34 pacientes $(54,8 \%), 16$ pacientes $(25,8 \%)$ requirieron curas de corticoides orales, 7 pacientes $(11,3 \%)$ visitas a urgencia por exacerbaciones y no se reportaron hospitalizaciones debidas al asma.

$\mathrm{Al}$ año de seguimiento disminuyó la proporción de pacientes con IOS alterada y aumentó la proporción de pacientes con RB+ (figura 1).

El 25,8\% (16 pacientes) de los que tenían IOS inicial alterada cambiaron a IOS normal en el segundo estudio, y solo 9,8\% (6 pacientes) que en el primer estudio registraron IOS normal cambiaron a IOS alterada en el 2do estudio, diferencia que fue significativa ( $\mathrm{p}=0,04)$. El 12,9\% (8 pacientes) de los que tenían $\mathrm{RB}+$ en el primer estudio cambiaron a RB- en el segundo estudio y el 19,4\% (12 pacientes) con RB- en el primer estudio cambiaron a RB+ en el segundo estudio, diferencia que no fue significativa $(\mathrm{p}=0,43)$.

Los promedios de los valores basales de $\mathrm{R} 5 \mathrm{Kpa} / \mathrm{Ls}$, R20 Kpa/Ls, X5 Kpa/Ls, AX Kpa/Ls y D5-20 Kpa/Ls mejoraron significativamente en la IOS final respecto de la inicial (tabla 2). Se midió la variación de los promedios de RB en estos mismos parámetros, sin encontrar diferencias significativas entre la IOS inicial y final (datos no incluidos). La tabla 3 muestra la variación de los promedios basales de la IOS final respecto de la inicial, entre asmáticos controlados y no controlados al final del seguimiento. En el grupo de controlados se encontró mejoría estadísticamente significativa en promedios basales de R5, R20, AX. En los no controlados, se encontró una mejoría estadísticamente significativa en todos los parámetros basales analizados (R5, R20, X5, AX y D5-20). Se realizó la medición de la variación de la RB+ en controlados y no controlados, sin encontrar diferencias significativas (datos no incluidos).

De los 62 pacientes, 47 realizaron el test cutáneo, y en este subgrupo se midió la variación de promedios basales y de respuesta broncodilatadora entre la IOS inicial y final. En este grupo encontramos cuatro categorías de respuesta oscilométrica al año de seguimiento: $6(12,7 \%)$ con TC negativo controlados, $13(27,7 \%)$ con TC negativo no controlados, $13(27,7 \%)$ con TC positivo controlados y $15(31,9 \%)$ con TC positivo no controlados (tabla 4). En ninguna de estas cuatro categorías analizadas se encontró variación de promedios de respuesta broncodilatadora estadísticamente significativa (datos no incluidos).

Tabla 1. Variables demográficas y resultados del test cutáneo en preescolares con asma

\begin{tabular}{lc}
\hline Variable & $\mathrm{n}(\%) \circ \overline{\mathrm{X}} \pm \mathrm{DS}$ \\
\hline Varones & $32(51,6)$ \\
Edad & $10(16,1)$ \\
3 años & $27(43,6)$ \\
4 años & $25(40,3)$ \\
5 años & $19,1 \pm 3,2$ \\
Peso $(\mathrm{kg})$ promedio & $110 \pm 5,9$ \\
Talla $(\mathrm{cm})$ promedio & $28(59,6)$ \\
Test cutáneo positivo &
\end{tabular}

$\overline{\mathrm{X}}$ : promedio o media aritmética; DS: desviación estándar.

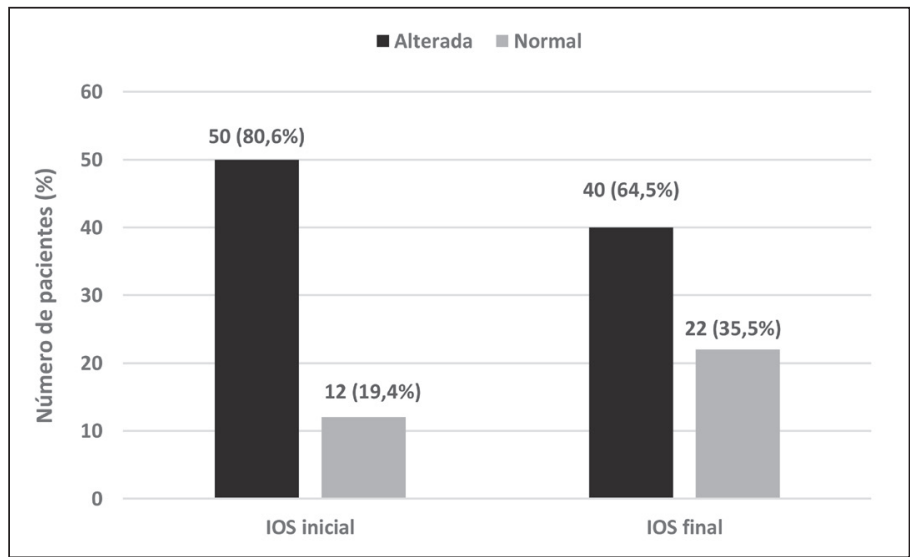

Figura 1. Número de pacientes y porcentaje con oscilometría de impulso (IOS) alterada en estudio inicial y tras un año de seguimiento.

Tabla 2. Análisis comparativo de parámetros de oscilometría de impulso iniciales tras un año de seguimiento en preescolares asmáticos

\begin{tabular}{lccl}
\hline Parámetro IOS & $\begin{array}{c}\text { IOS inicial } \\
\bar{X} \pm D S\end{array}$ & $\begin{array}{c}\text { IOS final } \\
\bar{X} \pm D S\end{array}$ & $p$ \\
\hline R5 (Kpa/Ls) & $1,01 \pm 0,19$ & $0,82 \pm 0,2$ & 0,00001 \\
R20 (Kpa/Ls) & $0,71 \pm 0,12$ & $0,56 \pm 0,12$ & 0,00001 \\
X5 (Kpa/Ls) & $-0,35 \pm 0,14$ & $-0,29 \pm 0,09$ & 0,0003 \\
AX (Kpa/Ls) & $3,22 \pm 1,5$ & $2,48 \pm 1,32$ & 0,00004 \\
D5-20 (Kpa/Ls) & $0,31 \pm 0,13$ & $0,26 \pm 0,13$ & 0,02 \\
\hline
\end{tabular}

R5: resistencia a 5 Hertz, R20: resistencia a 20 Hertz, X5: reactancia a 5 Hertz; AX: área de reactancia, D5-20: diferencia de resistencia a 5 y 20 Hertz, Kpa/Ls: kilopascal/litro por segundo, IOS: oscilometría de impulso, $\overline{\mathrm{X}}$ : promedio o media aritmética; DS: desviación estándar. 


\begin{tabular}{|c|c|c|c|c|c|c|}
\hline \multirow[b]{2}{*}{ Parámetro IOS } & \multicolumn{3}{|c|}{ Asma controlada $(n=28)$} & \multicolumn{3}{|c|}{ Asma no controlada $(n=34)$} \\
\hline & $\begin{array}{l}\text { IOS inicial } \\
\bar{x} \pm D S\end{array}$ & $\begin{array}{l}\text { IOS final } \\
\bar{X} \pm D S\end{array}$ & $\mathrm{p}$ & $\begin{array}{l}\text { IOS inicial } \\
\bar{x} \pm D S\end{array}$ & $\begin{array}{l}\text { IOS final } \\
\bar{X} \pm D S\end{array}$ & $\mathrm{p}$ \\
\hline R5 (Kpa/Ls) & $0,99 \pm 0,16$ & $0,82 \pm 0,17$ & 0,00001 & $1,04 \pm 0,21$ & $0,83 \pm 0,16$ & 0,00001 \\
\hline R20 (Kpa/Ls) & $0,7 \pm 0,11$ & $0,57 \pm 0,13$ & 0,00001 & $0,71 \pm 0,13$ & $0,56 \pm 0,12$ & 0,00001 \\
\hline X5 (Kpa/Ls) & $-0,34 \pm 0,19$ & $-0,28 \pm 0,08$ & NS & $-0,37 \pm 0,08$ & $-0,3 \pm 0,1$ & 0,001 \\
\hline $\mathrm{AX}(\mathrm{Kpa} / \mathrm{Ls})$ & $2,9 \pm 1,6$ & $2,3 \pm 1,2$ & 0,02 & $3,5 \pm 1,37$ & $2,7 \pm 1,38$ & 0,0007 \\
\hline D5-20 (Kpa/Ls) & $0,28 \pm 0,12$ & $0,25 \pm 0,13$ & NS & $0,33 \pm 0,14$ & $0,26 \pm 0,13$ & 0,04 \\
\hline
\end{tabular}

R5: resistencia a 5 Hertz; R20: resistencia a 20 Hertz; X5: reactancia a 5 Hertz; AX: área de reactancia; D5-20: diferencia de resistencia a 5 y 20 Hertz; Kpa/Ls: kilopascal/litro por segundo; IOS: oscilometría de impulso; $\bar{X}$ : promedio o media aritmética; DS: desviación estándar; NS: no significativo.

Tabla 4. Variación de parámetros de oscilometría de impulso tras un año de seguimiento en preescolares según resultado del test cutáneo y control del asma

\begin{tabular}{|c|c|c|c|c|c|c|c|c|}
\hline \multirow[b]{3}{*}{ Parámetro IOS } & \multicolumn{4}{|c|}{ Test cutáneo negativo } & \multicolumn{4}{|c|}{ Test cutáneo positivo } \\
\hline & \multicolumn{2}{|c|}{ Asma controlada } & \multicolumn{2}{|c|}{ Asma no controlada } & \multicolumn{2}{|c|}{ Asma controlada } & \multicolumn{2}{|c|}{ Asma no controlada } \\
\hline & $\begin{array}{l}\text { IOS Inicial } \\
\bar{x} \pm D S\end{array}$ & $\begin{array}{c}\text { IOS Final } \\
\bar{x} \pm D S\end{array}$ & $\begin{array}{l}\text { IOS Inicial } \\
\bar{x} \pm D S\end{array}$ & $\begin{array}{c}\text { IOS Final } \\
\bar{X} \pm D S\end{array}$ & $\begin{array}{l}\text { IOS Inicial } \\
\bar{x} \pm D S\end{array}$ & $\begin{array}{c}\text { IOS Final } \\
\bar{x} \pm D S\end{array}$ & $\begin{array}{l}\text { IOS Inicial } \\
\bar{X} \pm D S\end{array}$ & $\begin{array}{l}\text { IOS Final } \\
\bar{x} \pm D S\end{array}$ \\
\hline R5 (Kpa/Ls) & $1,02 \pm 0,2$ & $0,8 \pm 0,2^{*}$ & $1,08 \pm 0,2$ & $0,8 \pm 0,2 *$ & $0,98 \pm 0,1$ & $0,81 \pm 0,1 *$ & $1,07 \pm 0,1$ & $0,85 \pm 0,1$ * \\
\hline R20 (Kpa/Ls) & $0,7 \pm 0,1$ & $0,53 \pm 0,1$ & $0,72 \pm 0,1$ & $0,51 \pm 0,1 *$ & $0,73 \pm 0,1$ & $0,57 \pm 0,1$ * & $0,73 \pm 0,1$ & $0,59 \pm 0,1$ * \\
\hline X5 (Kpa/Ls) & $-0,46 \pm 0,4$ & $-0,29 \pm 0,1$ & $-0,4 \pm 0,1$ & $-0,34 \pm 0,1$ & $-0,3 \pm 0,08$ & $-0,28 \pm 0,07$ & $-0,37 \pm 0,06$ & $-0,28 \pm 0,06$ * \\
\hline $\mathrm{AX}(\mathrm{Kpa} / \mathrm{Ls})$ & $3,5 \pm 3,3$ & $2,6 \pm 1,8$ & $4 \pm 1,4$ & $3 \pm 1,4^{*}$ & $2,5 \pm 0,6$ & $2,2 \pm 1,1$ & $3,4 \pm 1,3$ & $2,5 \pm 1,4$ * \\
\hline D5-20 (Kpa/Ls) & $0,3 \pm 0,2$ & $0,3 \pm 0,1$ & $0,35 \pm 0,1$ & $0,33 \pm 0,1$ & $0,25 \pm 0,1$ & $0,24 \pm 0,1$ & $0,34 \pm 0,1$ & $0,25 \pm 0,1$ * \\
\hline
\end{tabular}

R5: resistencia a 5 Hertz, R20: resistencia a 20 Hertz, X5: reactancia a 5 Hertz, AX: área de reactancia, D5-20: diferencia de resistencia a 5 y 20 Hertz, Kpa/Ls: kilopascal/litro por segundo, IOS: oscilometría de impulso, $\overline{\mathrm{X}}$ : promedio o media aritmética, DS: desviación estándar, * $p<0,05$.

\section{Discusión}

Nuestros resultados muestran que la IOS detecta alteraciones en la función pulmonar en prescolares asmáticos, lo que concuerda con lo publicado por distintos autores, que indican que es una prueba valiosa para evaluar la función pulmonar y hacer el diagnóstico de asma a corta edad ${ }^{8,9,20,21}$. Al terminar el seguimiento se encontró mejoría significativa de IOS previamente alterada, sin embargo un alto porcentaje mantuvo alteraciones, lo que indicaría que en prescolares asmáticos, con las características clínicas del grupo estudiado, la mejoría es lenta, pudiendo ser explicada por el tratamiento usado o la severidad de la enfermedad, manifestada por un frecuente compromiso de la función de vía aérea pequeña. De hecho, Shi et al. demostraron que parámetros de función la vía aérea pequeña como la D5-20 y AX, fueron útiles en discriminar entre controlados de no controlados y predecir el riesgo de pérdida del control del asma en un seguimiento de 3 meses de niños asmáticos ${ }^{13}$. La mejoría de la IOS observada a lo largo de un año coincide con lo comunicado por Saadeh, quien encontró que los niños asmáticos en tratamiento permanente para el asma presentan una mejoría en R5 y AX a lo largo del tiempo ${ }^{14}$. El valor promedio de todos los parámetros de la IOS mejora significativamente después de un año de tratamiento, lo que podría reflejar que un grupo importante logra mejorar su función pulmonar. Sin embargo, alrededor del 50\% mantuvo asma no controlada y la mayoría persistió con tratamiento controlador. Esto se podría explicar por mala adherencia al tratamiento, que este haya sido subóptimo, o que este sea un grupo de asmáticos de mayor severidad, que a nuestro juicio es lo más probable. Esto último, se podría inferir debido al tipo de alteraciones encontradas en la IOS, y que concuerda con un estudio previo realizado en preescolares asmáticos, en que se demostró que algunos de los parámetros de la IOS son útiles para medir la severidad de la enfermedad ${ }^{22}$. 
El grupo de los no controlados registró mejoría significativa en todas las variables, sin embargo, en los controlados no hubo mejoría en X5 ni D5-20. Pensamos que esto se debe a que el grupo de no controlados inició el seguimiento con mayores promedios en $\mathrm{AX}$ y D5-20 y menores en X5, es decir con mayor compromiso de la vía aérea fina que los controlados. Esto concuerda con algunos estudios en que estos parámetros han sido reportados como buenos predictores de control o exacerbaciones a mediano plazo ${ }^{11,13,23}$. También existe evidencia reciente que demuestra que la alteración de parámetros de vía aérea periférica en IOS se podría encontrar hasta en el 20\% de los de los asmáticos menores de doce años con buen control de la enfermedad, lo que podría determinaría un nuevo fenotipo de asma, con implicancias clínicas y pronóstico aún desconocidos ${ }^{24}$.

En preescolares con asma, se ha postulado que la $\mathrm{RB}+$ en la IOS podría identificar la hiperreactividad bronquial de la pequeña vía aérea con mayor exactitud que la espirometría, contribuyendo a una mejor caracterización fisiopatológica y clínica de la enfermedad ${ }^{25}$.

Un hallazgo inesperado en este estudio fue el aumento de la RB+ en la IOS final respecto de la inicial y la ausencia de disminución significativa en los promedios de los parámetros individuales de $\mathrm{RB}+$ entre ambos estudios. Esto podría explicarse por la severidad del asma de los niños incluidos, quienes mantienen la hiperreactividad de la vía aérea, hecho que se ha reportado en estudios se seguimiento a largo plazo con preescolares asmáticos en que la persistencia de la RB+ se asocia a mayor respuesta broncodilatadora en la adolescencia, lo que justificaría su seguimiento ${ }^{26}$.

Se encontró cuatro diferentes tipos de evolución en los parámetros oscilométricos según el control del asma y la sensibilización alérgica, los cuales podrían representar distintos fenotipos de asma del prescolar. El grupo de pacientes no atópicos y que no estaban controlados se registraron variaciones significativas en los promedios de R20 y AX, que no estuvieron presentes en los controlados. En el grupo de atópicos que no estaban controlados las variaciones significativas de promedios no encontradas en los controlados ocurrieron en X5, AX y D5-20. Postulamos a que el grupo no atópico controlado podría corresponder a niños menos severos, que dejarán de tener sibilancias, mientras que los atópicos no controlados tendrían una enfermedad más severa y con mayor disfunción de la vía aérea periférica. En este último grupo se ha reportado mayor riesgo de persistir con asma y mejor respuesta a la terapia con esteroides inhalados lo que, a nuestro parecer, podría explicar la mejoría estadísticamente significativa encontrada de todos los promedios de parámetros de la IOS luego de un año de tratamiento ${ }^{27,28}$. Esto coincide con lo informado por
Reddy y cols., quienes pudieron distinguir distintos fenotipos de prescolares sibilantes usando la IOS, entre ellos sibilantes severos atópicos y no atópicos. En ambos grupos hubo disfunción de la vía aérea evaluada por IOS y se caracterizaron por consultas a urgencia, exacerbaciones, curas esteroidales pese a estar en tratamiento controlador ${ }^{29}$. Los autores recalcan la importancia de identificar precozmente estos fenotipos, y sugieren que sus hallazgos podrían estar alineados con otro estudio que logró identificar a dos grupos de asmáticos escolares con altos requerimientos de terapia controladora, uno atópico con función pulmonar moderadamente disminuida más exacerbaciones frecuentes y otro no atópico con función pulmonar levemente disminuida, menos exacerbaciones, pero altamente sintomático ${ }^{30}$.

De todos modos, creemos que el hallazgo de IOS alterada en asma preescolar, justifica el seguimiento a largo plazo en todos los grupos, independientes de su fenotipo, ya que hay evidencia que ha demostrado que las alteraciones de la función pulmonar a corta edad se asocian a mayor necesidad de tratamiento, persistencia de síntomas y de alteraciones de la función pulmonar en la adolescencia ${ }^{26,31,32}$.

Nuestros resultados nos permiten afirmar que la IOS es un estudio útil en seguimiento y monitoreo del asma en preescolares, su uso, detecta disfunción de la vía aérea pequeña y podría ayudar a evaluar mejor el efecto de distintos tratamientos (ej. preferir los esteroides ultrafinos que actúan en las vías respiratorias periféricas) y distinguir posibles diferentes fenotipos de asma a esta edad.

La limitación más importante del estudio es el bajo número de pacientes incluidos, lo que le restó potencia a las comparaciones entre atópicos y no atópicos. Un número mayor de pacientes podría permitir establecer un valor predictivo para algunos parámetros de la IOS, caracterizar mejor la función de la vía aérea pequeña, su relación con tener un mal control o persistencia del asma y el rol de la atopia. Se necesitan estudios adicionales de función pulmonar en el asma que incluyan asmáticos episódicos e intermitentes leves a moderados a más largo plazo y que además permitan evaluar el impacto de distintas terapias en su evolución.

En conclusión, nuestros hallazgos confirman la IOS de niños en etapa preescolares mejora significativamente a un año de seguimiento, sin embargo, estos niños mantienen un porcentaje elevado de IOS alterada y $\mathrm{RB}+$ que podría reflejar la severidad de la enfermedad. La variación de parámetros que reflejan la obstrucción de la vía aérea pequeña podría ser útil en identificar y caracterizar algunos fenotipos de asma recientemente reportados, que se han asociado a mayor severidad y persistencia de síntomas hasta la adolescencia. 


\section{Responsabilidades Éticas}

Protección de personas y animales: Los autores declaran que los procedimientos seguidos se conformaron a las normas éticas del comité de experimentación humana responsable y de acuerdo con la Asociación Médica Mundial y la Declaración de Helsinki.

Confidencialidad de los datos: Los autores declaran que han seguido los protocolos de su centro de trabajo sobre la publicación de datos de pacientes.
Derecho a la privacidad y consentimiento informado: Los autores han obtenido el consentimiento informado de los pacientes y/o sujetos referidos en el artículo. Este documento obra en poder del autor de correspondencia.

\section{Conflicto de intereses}

Los autores declaran no tener conflicto de intereses.

\section{Referencias}

1. Bacharier L. The recurrently wheezing preschool child-benign or asthma in the making? Ann Allergy, Asthma Immunol. 2015;115(6):463-70.

2. Grad R, Morgan W. Long-term outcomes of early-onset wheeze and asthma. J Allergy Clin Immunol. 2012;130(2):299307.

3. Guerra S, Martínez F. Epidemiology of the Origins of Airflow Limitation in Asthma. Proc Am Thorac Soc. 2009;6(8):707-11.

4. Martinez F. The Origins of Asthma and Chronic Obstructive Pulmonary Disease in Early Life. Proc Am Thorac Soc. 2009;6(3):272-77.

5. Beydon N, Davis S, Lombardi E, et al. An official American Thoracic Society/ European Respiratory Society statement: pulmonary function testing in preschool children. Am J Respir Crit Care Med. 2007;175(12):1304-45.

6. Gaffin J, Shotola N, Martin T, Phipatanakul W. Clinically useful spirometry in preschool-aged children: evaluation of the 2007 American Thoracic Society Guidelines. J Asthma. 2010;47(7):762-7.

7. Marotta A, Klinnert M, Price M, Larsen G, Liu A. Impulse oscillometry provides an effective measure of lung dysfunction in 4-year-old children at risk for persistent asthma. J Allergy Clin Immunol. 2003;112(2):317-22.

8. Bickel S, Popler J, Lesnick B, Eid N. Impulse oscillometry: interpretation and practical applications. Chest. 2014;146(3):841-47.

9. Knihtilä H, Kotaniemi-Syrjänen A, Pelkonen A, Kalliola S, Mäkelä M, Malmberg L. Small airway oscillometry indices: Repeatability and bronchodilator responsiveness in young children. Pediatr Pulmonol. 2017;52(10):1260-67.

10. Komarow H, Skinner J, Young M, et al. A study of the use of impulse oscillometry in the evaluation of children with asthma: analysis of lung parameters, order effect, and utility compared with spirometry.
Pediatr Pulmonol. 2012;47(1):18-26.

11. Shi Y, Aledia A, Tatavoosian A, Vijayalakshmi S, Galant S, George S. Relating small airways to asthma control by using impulse oscillometry in children. J Allergy Clin Immunol. 2012;129(3):6718.

12. Schulze J, Biedebach S, Christmann M, Herrmann E, Voss S, Zielen S. Impulse Oscillometry as a Predictor of Asthma Exacerbations in Young Children. Respiration. 2016;91(2):107-14.

13. Shi Y, Aledia A, Galant S, George S. Peripheral airway impairment measured by oscillometry predicts loss of asthma control in children. J Allergy Clin Immunol. 2013;131(3):718-23.

14. Saadeh C, Cross B, Saadeh C, Gaylor $M$. Retrospective observations on the ability to diagnose and manage patients with asthma through the use of impulse oscillometry: comparison with spirometry and overview of the literature Pulm Med. 2014:376890; https://doi. org/10.1155/2014/376890, última visita 03 de abril de 2020 .

15. Shin Y, Jang S, Yoon J, et al. Oscillometric and spirometric bronchodilator response in preschool children with and without asthma. Can Respir J. 2012;19(4):273-7.

16. Meraz E, Nazeran H, Ramos C, et al. Analysis of impulse oscillometric measures of lung function and respiratory system model parameters in small airway-impaired and healthy children over a 2-year period. Biomed Eng Online. 2011;10:21; https://doi.org/10.1186/1475925X-10-21, última visita 03 de abril de 2020.

17. Global Initiative for Asthma. Global Strategy for Asthma Management and Prevention 2016: www.Ginasthama.org. última visita 3 de abril de 2020 .

18. Gochicoa-Rangel L, Cantú-González G, Miguel-Reyes JL, Luis Rodríguez-Moreno L, Torre-Bouscoulet L. Oscilometría de impulso. Recomendaciones y procedimiento. Neumol Cir Torax 2014;73(2):138-49.

19. Duiverman E, Clément J, van de
Woestijne K, Neijens H, van den Bergh A, Kerrebijn K. Forced oscillation technique. Reference values for resistance and reactance over a frequency spectrum of 2-26 Hz in healthy children aged 2.3-12.5 years. Bull Eur Physiopathol Respir. 1985;21(2):171-8.

20. Galant S, Komarow H, Shin H, Siddiqui $S$, Lipworth B. The case for impulse oscillometry in the management of asthma in children and adults. Ann Allergy Asthma Immunol. 2017;118(6):664-71.

21. Song T, Kim K, Kim E, Park J, Sohn M, Kim K. Utility of impulse oscillometry in young children with asthma. Pediatr Allergy Immunol. 2008;19(8):763-8.

22. Shin Y, Yoon J, Choi S, et al. Use of impulse oscillometry system in assessment of asthma severity for preschool children. J Asthma. 2013;50(2):198-203.

23. Zeng J, Chen Z, Hu Y, Hu Q, Zhong S, Liao W. Asthma control in preschool children with small airway function as measured by IOS and fractional exhaled nitric oxide. Respir Med. 2018;145:8-13.

24. Tirakitsoontorn P, Crookes M, Fregeau $\mathrm{W}$, et al. Recognition of the peripheral airway impairment phenotype in children with well-controlled asthma. Ann Allergy Asthma Immunol. 2018;121(6):692-98.

25. Sheen $Y$, Jee H, Ha E, et al. Impulse oscillometry and spirometry exhibit different features of lung function in bronchodilation. J Asthma. 2018;55(12):1343-51.

26. Lauhkonen E, Riikonen R, Törmänen S. Impulse oscillometry at preschool age is a strong predictor of lung function by flow-volume spirometry in adolescence. Pediatr Pulmonol. 2018;53(5):552-8.

27. Bacharier L, Guilbert $\mathrm{T}$, Zeiger R, et al. Patient characteristics associated with improved outcomes with use of an inhaled corticosteroid in preschool children at risk for asthma. J Allergy Clin Immunol. 2009;123(5):1077-82.

28. Fitzpatrick A, Bacharier L, Guilbert T, et al. Phenotypes of Recurrent Wheezing 
in Preschool Children: Identification by Latent Class Analysis and Utility in Prediction of Future Exacerbation. J Allergy Clin Immunol Pract. 2019;7(3):915-24.

29. Reddy M, Liu A, Robinson J, Klinnert M. Recurrent wheeze phenotypes in poor urban preschool-age children. J Allergy
Clin Immunol Pract. 2019;7(2):736-39.

30. Zoratti E, Krouse R, Babineau D, et al. Asthma phenotypes in inner-city children. J Allergy Clin Immunol. 2016;138(4):1016-29.

31. Knihtilä H, Kotaniemi-Syrjänen A, Mäkelä M, et al. Bondestam J, Pelkonen AS, Malmberg LP. Preschool oscillometry and lung function at adolescence in asthmatic children. Pediatr Pulmonol. 2015;50(12):1205-13.

32. Lajunen K, Kalliola S, KotaniemiSyrjanenen A, et al. Abnormal lung finction at preschool age asthma in adolescence? Ann Allergy Asthma Immunol 2018;120:520-26. 